\title{
Characterizing Multi-layer Pristine Graphene, Its Contaminants, and Their Origin Using Transmission Electron Microscopy
}

\author{
Todd H. Brintlinger, ${ }^{1}$ Nabil D. Bassim, ${ }^{1 *}$ Jonathan Winterstein, ${ }^{1}$ Amy Ng, ${ }^{4}$ M.S. Lodge, ${ }^{5}$
}

Masahiro Ishigami, ${ }^{5}$ Keith Whitener, ${ }^{3}$ Paul Sheehan, ${ }^{3}$ Rhonda M. Stroud, ${ }^{1}$ and Jeremy T. Robinson ${ }^{2}$

1. Materials Sci. and Tech. Division, U.S. Naval Research Laboratory, Washington, DC, USA 20375

2. Electronics Sci. and Tech. Division, U.S. Naval Research Laboratory, Washington, DC, USA 20375

3. Chemistry Division, U.S. Naval Research Laboratory, Washington, DC, USA 20375

4. NRC Postdoctoral Associate, U.S. Naval Research Laboratory, Washington, DC, USA 20375

5. Dept. of Physics and Nanoscience Technology Ctr., Univ. of Central Florida, Orlando, FL USA 32816

*current address: Dept. of Mat. Sci. and Eng., McMaster Univ., Hamilton, Ontario, Canada L9H 4L7

Graphene is often presented schematically as one or more continuous sheet(s) of planar carbon atoms. In reality the sheets are not planar[1,2] and have contaminants, particularly disordered carbonaceous materials, which are introduced during growth, handling, and transfer to specific substrates. To increase the area of pristine graphene, this contamination must be characterized accurately and reproducibly enough to provide a metric for improvements within a given 'lot' of graphene or graphene-based heterostructures on a specific substrate. Since Raman microscopy is relatively insensitive to disordered carbon, and very thin layers might be overlooked by high-resolution transmission electron microscopy (HRTEM), aberration-corrected scanning transmission electron microscopes (ac-STEMs) are the most reliable tools to confirm and quantify the presence of this contamination. Here, we use a Nion UltraSTEM200-X to investigate the characteristics and behavior of the contamination layer on different substrates, with different transfer techniques, and with different growth parameters. In Fig. 1, we compare contamination on two samples from the same graphene growth (chemical vapor deposition on copper), but with different transfer techniques to place the suspended graphene on holey carbon TEM grids. The left panel is transferred using our technique for retaining chemical functionality with the Birch reduction of graphene that uses neither a polymer support nor a copper etchant [3]. The right panel is a conventional PMMA-based transfer which showed larger areas of pristine graphene, a surprising result since polymer residue and hydrocarbon contamination are thought to impact ultimate cleanliness. Further work revealed that different transfer techniques yield contamination of similar distribution and morphology despite post-transfer modification by plasma cleaning, ex situ annealing and in situ selfheating. Furthermore, changing growth parameters affects the disordered carbon on the sample, suggesting contamination may originate during the growth of graphene itself and not in subsequent handling steps.

Despite their utility, ac-STEMs are expensive and time-consuming, which limits the availability and rapidity of this characterization. We also show here that HRTEM can be used to accurately characterize multi-layer graphene and its contaminants, as seen in Fig. 2. A comparison between this data with previous ac-STEM measurements allows us to use both to understand the origin of contaminants on astransferred, chemical-vapor-deposition grown, suspended multi-layer graphene on different substrates.

References:

[1] Meyer et al Nature 446 (2007), p 60.

[2] Ishigami et al Nano Lett. 7 (2007), p 1643.

[3] Whitener et al Nano Lett. 16 (2016), p 1455. 

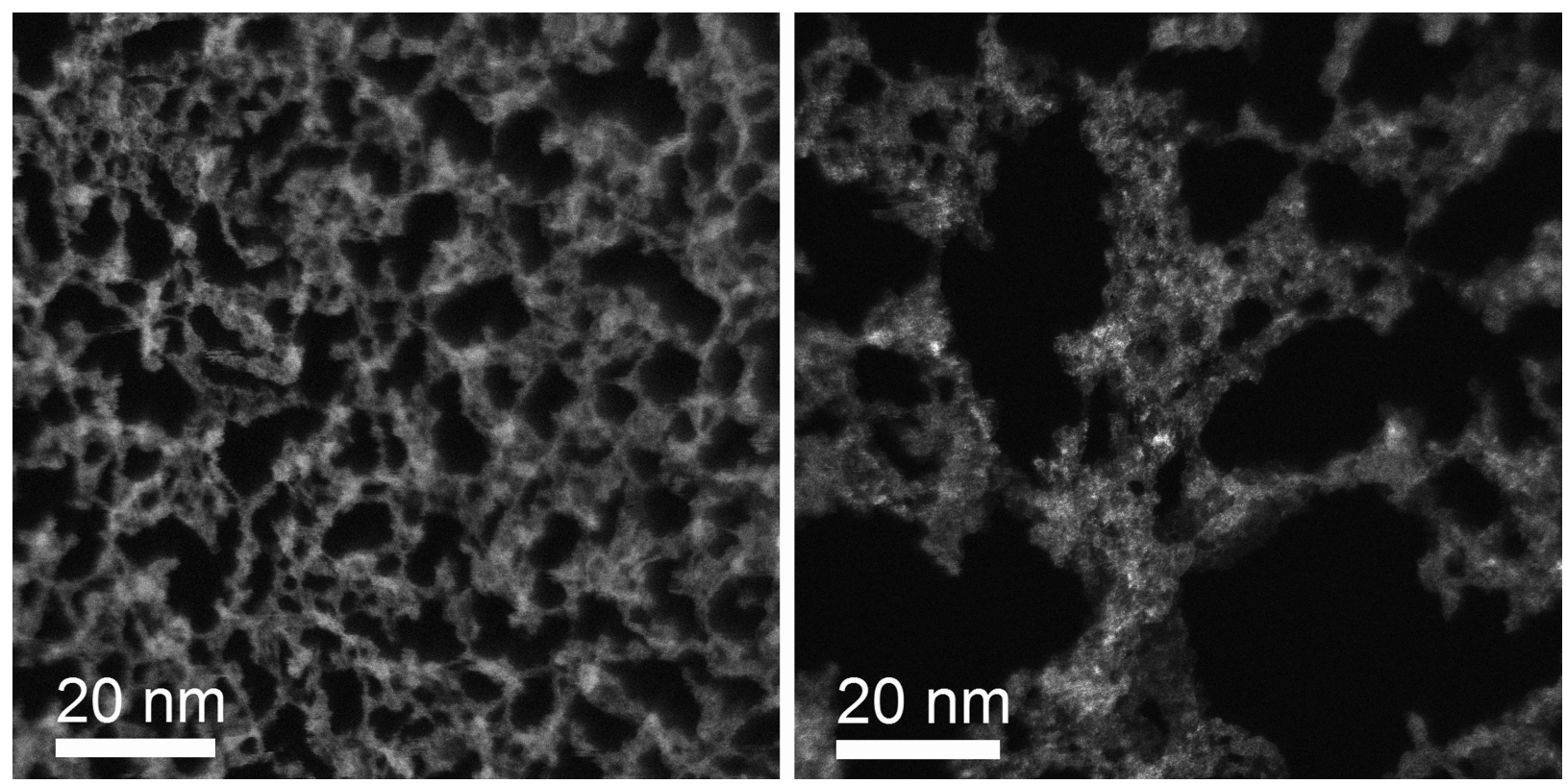

Figure 1: Aberration-corrected scanning transmission electron microscopy in medium angle annular dark field (STEM-MAADF) of the same starting graphene transferred to holey carbon TEM grids using: (left) water-based polymer- and etchant-free transfer to preserve chemical modifications following Birch reduction of graphene, (right) standard PMMA-based transfer. Dark areas are pristine graphene.
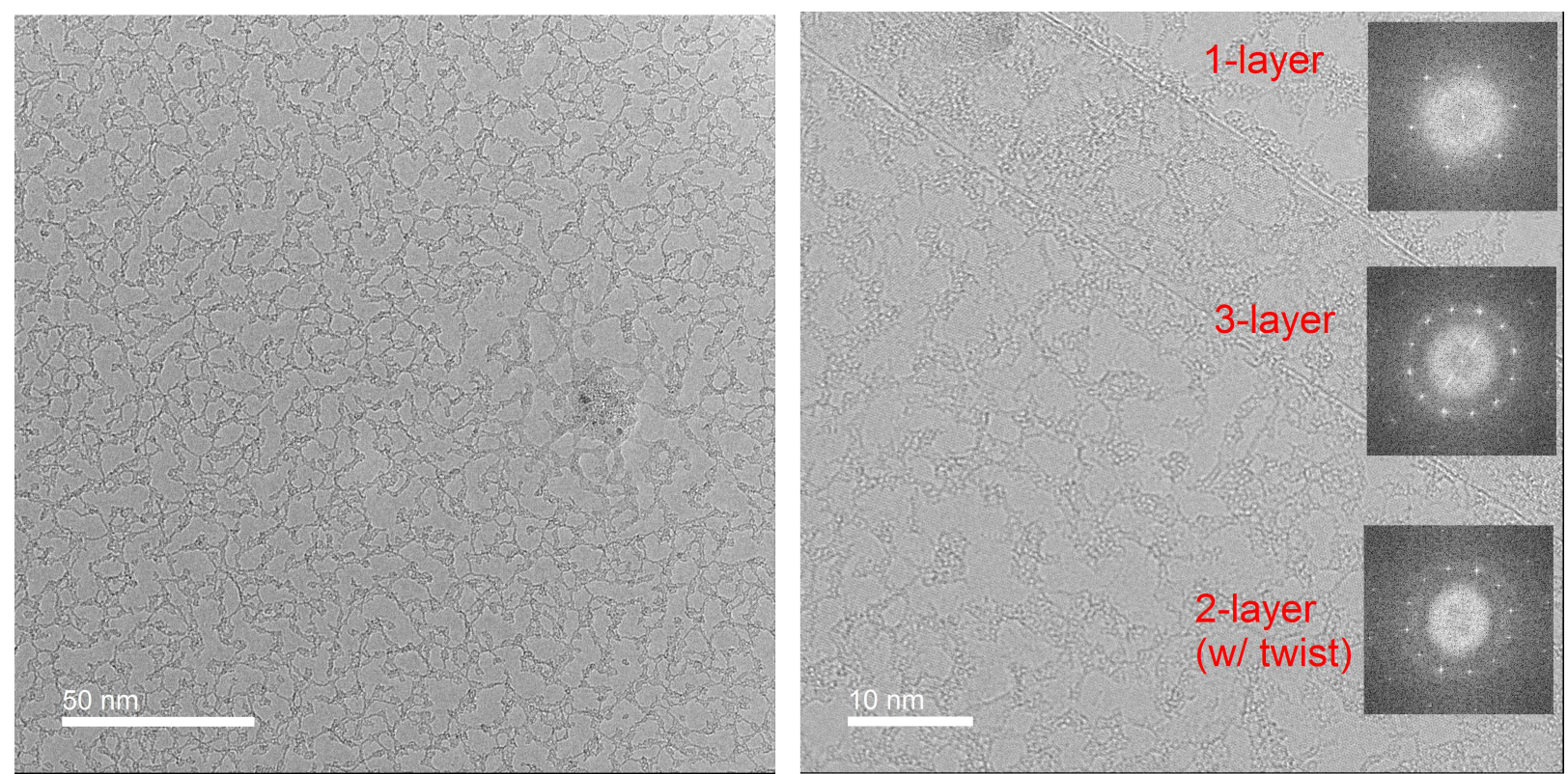

Figure 2: High-resolution transmission electron microscopy (HRTEM) with an uncorrected microscope $\left(C_{s}=0.5 \mathrm{~mm}\right)$ of same graphene as seen in left panel of Fig. 1. Left panel is large area overview showing distribution of contamination, which is similar to STEM-MAADF. Right panel is higher magnification micrograph with three reduced area Fast-Fourier-Transform (FFT) images from the three labeled regions: a single-layer region, a three-layer region that combines the single and bilayer regions, and a bilayer region (in which FFT shows the twist angle). Outer spots of FFT are $0.12 \mathrm{~nm}$. 